\title{
BMJ Open Trends and risk factors for HIV, HCV and syphilis seroconversion among drug users in a methadone maintenance treatment programme in China: a 7-year retrospective cohort study
}

Xia Zou, ${ }^{1,2}$ Li Ling, ${ }^{1,2}$ Lei Zhang ${ }^{2,3}$

To cite: Zou X, Ling L, Zhang $L$. Trends and risk factors for HIV, HCV and syphilis seroconversion among drug users in a methadone maintenance treatment programme in China: a 7-year retrospective cohort study. BMJ Open 2015;5:e008162.

doi:10.1136/bmjopen-2015008162

\section{- Prepublication history and additional material is available. To view please visit the journal (http://dx.doi.org/ 10.1136/bmjopen-2015- 008162)}

Received 11 March 2015 Revised 16 June 2015 Accepted 14 July 2015

\section{CrossMark}

${ }^{1}$ Faculty of Medical Statistics and Epidemiology, School of Public Health, Sun Yat-sen University, Guangzhou, People's Republic of China ${ }^{2}$ Sun Yat-sen Centre for Migrant Health Policy, Sun Yat-sen University, Guangzhou, People's Republic of China ${ }^{3}$ The Kirby Institute, University of New South Wales, Sydney, New South Wales, Australia

\section{Correspondence to}

Li Ling; lingli@mail.sysu.edu.cn and Lei Zhang;

Izhang@kirby.unsw.edu.au

\section{ABSTRACT}

Objective: This study explores the trends and associated factors of HIV, hepatitis $\mathrm{C}$ virus (HCV) and syphilis seroconversion among Chinese methadone maintenance treatment (MMT) clients over a follow-up period of up to 7 years.

Design: Drug users from 14 MMT clinics in Guangdong Province were recruited during 2006-2014. Participants were seronegative with at least one HIV, HCV or syphilis infection at baseline and had completed at least one follow-up test during the study period. We estimated HIV, HCV and syphilis seroconversion rates in follow-up years and identified the underlying predictors using a multivariate Cox regression model.

Results: Among 9240 participants, the overall HIV seroconversion rate was 0.20 (0.13 to 0.28$) / 100$ person-years (pys), 20.54 (18.62 to 22.46)/100 pys for HCV and 0.77 (0.62 to 0.93$) / 100$ pys for syphilis, over the study period. HIV seroconversion rate showed a moderate but non-significant annual decline of $13.34 \%$ $(-42.48 \%$ to $30.56 \%)$ ( $\chi^{2}$ trend test; $p=0.369$ ), whereas the decline of HCV seroconversion was $16.12 \%$ (5.53\% to $25.52 \%)$ per annum $(p<0.001)$. Syphilis seroconversion rate remained stable $(p=0.540)$. Urine results positive for opioid predicted HIV seroconversion ( $\geq 60 \%$ vs $<60 \%$; HR=3.40, 1.07 to 10.85 ), being unmarried ( $H R=1.59,1.15$ to 2.20), injection drug use in the past 30 days (HR=2.17, 1.42 to 3.32 ), having sexual intercourse in the past 3 months ( $H R=1.74,1.22$ to 2.47) and higher daily dosage of methadone ( $\geq 60 \mathrm{~mL}$ vs $<60 \mathrm{~mL} ; \mathrm{HR}=1.40,1.01$ to 1.94 ) predicted $\mathrm{HCV}$ seroconversion. Being female (HR=3.56, 2.25 to 5.64) and infected with HCV at baseline (HR=2.40, 1.38 to 8.36) were associated with subsequent syphilis seroconversion.

Conclusions: MMT in China has demonstrated moderate-to-good effectiveness in reducing HIV and HCV incidence but not syphilis infection among participating drug users.

\section{INTRODUCTION}

The rapid spread of blood-borne and sexually transmitted infections represents a major

\section{Strengths and limitations of this study}

- This study tested 4536, 1077 and 3910 samples for HIV, hepatitis C virus (HCV) and syphilis, respectively, and identified 27,440 and 98 persontimes seroconversions, respectively. However, national data with a larger sample size are needed.

- The participants in this cohort study were followed for 7 years, thus we were able to explore the trends of HIV, HCV and syphilis seroconversion rate by follow-up years.

- This study identified significant treatment-related factors that predicted HIV (positive rate for urine tests) and HCV (average dosage) seroconversions.

public health concern in many parts of the world. In particular, the transmission of HIV, hepatitis $\mathrm{C}$ virus (HCV) and syphilis infections has contributed to high levels of disease burden and mortality worldwide. Sharing of injection equipment for illicit drug use has fuelled the transmission of HIV and HCV in many developing country settings. ${ }^{1-3}$ Syphilis infection is commonly reported among drug users as illicit drug use also facilitates risky sexual behaviour. ${ }^{4}$ Opioid substitution therapy (OST) has been widely accepted as an effective harm reduction strategy for drug users. Accumulating evidence has shown that OST can effectively reduce risky drug-use behaviours ${ }^{5}$ and hence the risk of acquiring HIV and HCV among drug users.

Methadone maintenance treatment (MMT), a key component of OST, was initiated in China during 2004. As of 2013, 761 MMT clinics have cumulatively been established nationwide, linking over 2.47 million opioid drug users to the programme in China. ${ }^{6}$ The programme has shown to be effective in reducing risk behaviour among participating 
Chinese drug users. ${ }^{7}$ We established our study sites in Guangdong Province, an open and economically developed southern Chinese province with 370000 registered drug users. HIV, HCV and syphilis prevalence among drug users in Guangdong were reportedly as high as $6.3 \%$, $78.7 \%$ and $12.6 \%$, respectively. MMT in Guangdong was initiated in 2005. By the end of 2013, 60 MMT clinics had been established and cumulatively 30410 drug users had received MMT.

Previous studies have shown lower HIV and HCV incidence rates among MMT clients than among the community of drug users. ${ }^{8}$ Participants who have remained in MMT longer have also demonstrated lower risk in acquiring HIV, HCV and syphilis infections. ${ }^{10}{ }^{11}$ Numerous studies have reported the prevalence of HIV, HCV and syphilis in various subgroups of MMT clients and at different treatment stages. ${ }^{10-13}$ By linking infection status to clients' demographic, biological, behavioural and treatment-related indicators, these studies have provided important insights into the contributing factors of these infections at a certain time. ${ }^{14-16}$ However, infection prevalence is a good indication of disease burden but not of the trend of disease progression. Similarly, factors associated with disease prevalence explain the potential causes of infections at one fixed time but may not reflect the potential changes in risk factors due to treatment. Built on the previous findings, this study further explores the trends of HIV, HCV and syphilis seroconversion among MMT clients during the course of treatment, and identifies associated factors of new infections based on a retrospective cohort approach.

\section{METHODS}

\section{Study sites and participants}

Our study was conducted in 14 MMT clinics located in nine cities in Guangdong Province, China. This retrospective cohort study recruited 9412 MMT clients between July 2006 and March 2014. Participants were eligible if they were: (1) diagnosed with opioid dependence according to the International Classification of Diseases, 10th Revision, (2) aged 20 years or above and (3) local residents who settled in the catchment areas of the clinics. Written informed consent was obtained at treatment enrolment. We excluded participants who were (1) not tested for HIV, HCV and syphilis, (2) seropositive for HIV, HCV or syphilis at enrolment in their respective cohorts; however, clients infected with a different infection (eg, HCV) were allowed to enrol into other cohorts (eg, HIV and syphilis cohorts) and (3) those who did not complete at least one follow-up test. Three retrospective cohorts were created at baseline with regard to the negative infection status of HIV, HCV and syphilis, respectively (figure 1).

\section{Data collection}

Data were routinely collected and stored in the national unified MMT management system. At enrolment, demographic information was collected. Drug use-related behaviours and sexual behaviours were surveyed at both enrolment and subsequent half-yearly follow-ups. Blood samples of clients were also collected to assess HIV, hepatitis C and syphilis status at enrolment and follow-ups. Urine-opiate tests were performed at a random day each month. Methadone was dispensed to patients daily. All information was entered into the MMT management system by clinic staff. For this study, data of 14 studied clinics were extracted from the MMT management system, with personally identifiable information being removed.

\section{Laboratory tests}

Blood specimens were tested for HIV-1/2, HCV and syphilis. The presence of HIV antibodies was screened using a colloidal gold method (Aibo Biotech Company, China) in the MMT clinics. Positive blood specimens were sent to the provincial Center for Disease Control and Prevention for confirmatory tests. HIV infection was determined by two positive ELISAs (ELISA-1, Beijing BGI-GBI Biotech Company, Ltd, China; ELISA-2, the 4th generation ELISA, bioMerieux bv, The Netherlands) and western blot (Abbott, MP Biomedicals, LLC, Singapore). HCV testing was carried out using ELISAs (Aibo Biotech Company, China). Toluidine red unheated serum test (TRUST, Rongsheng Biotech Company, China) was performed on all serum samples. Confirmation of positive results was made with a Treponema palladium particle agglutination (TPPA, Lizhu Reagent Company, China) test.

MMT clients who were diagnosed with HIV, HCV and syphilis were notified and referred to designated public hospitals for further treatment.

\section{Statistical analysis}

The rate of positive urine tests is defined as the proportion of urine samples tested positive for opioids among the total urine samples submitted. Average MMT dosage is calculated by dividing the total dosage consumed by an individual by the number of days of attendance. Two-sample Student $t$ test, Mann-Whitney test and $\chi^{2}$ test were used for normally distributed continuous data, non-parametric data and frequencies, respectively.

The event of interest in this study was HIV, HCV and syphilis seroconversion during the course of MMT. For syphilis, an occasion of seroconversion was considered as a case with regard to the curability of the disease. The duration of follow-up was determined as the time between the date of MMT enrolment and the date of seroconversion, or the end of study. The date of seroconversion was approximated as the midpoint between the last negative and the first positive test for all infections. Seroconversion rate with $95 \%$ CIs was calculated as numbers of events over person-years, and its temporal trend significance was determined with a Joinpoint regression model. ${ }^{17}$ Univariate and multivariate Cox proportional hazard regression analyses were conducted to 


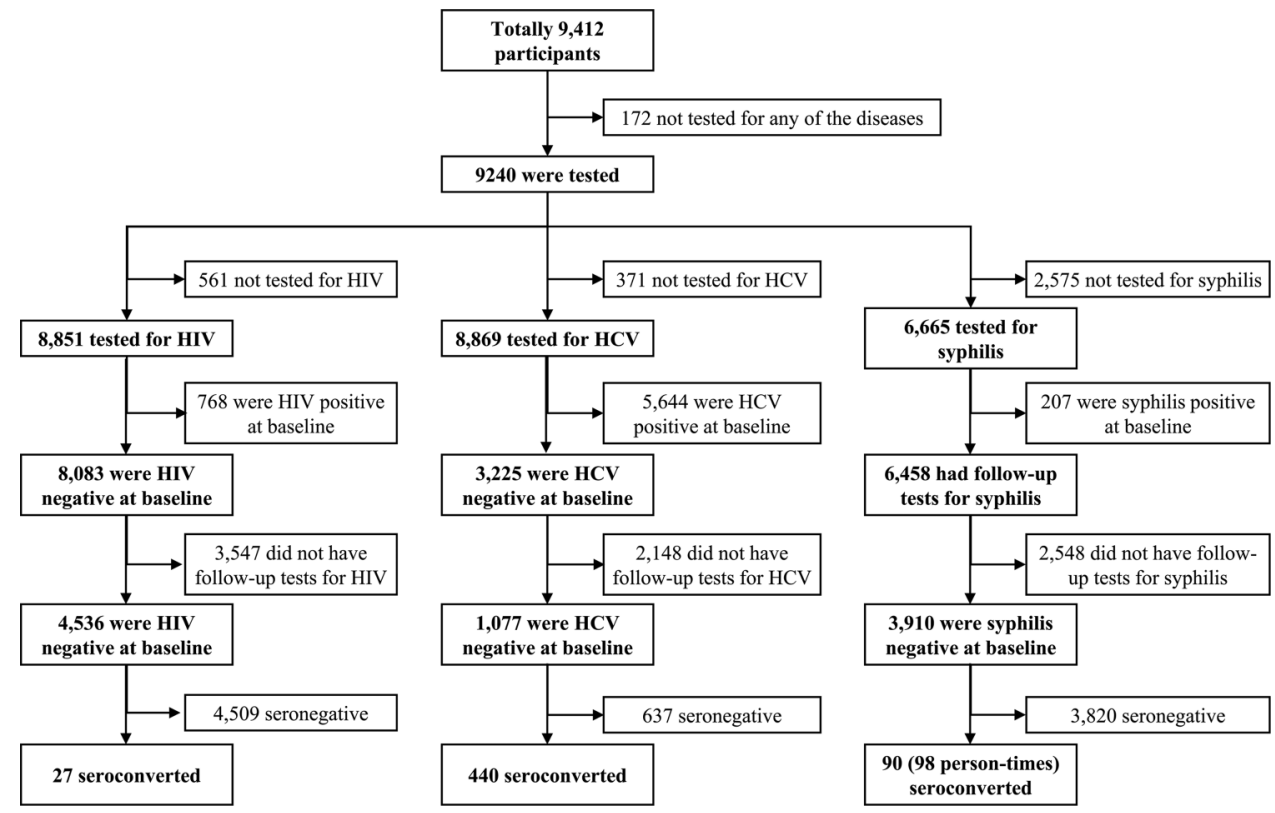

Figure 1 Eligibility and screening procedures for study participants (HCV, hepatitis C virus).

identify predictors of HIV, HCV and syphilis seroconversion. Variables with a bivariate $\mathrm{p}$ value $<0.20$ were included in subsequent multivariate regression. The last behavioural record before seroconversion or end point of the study was used for the regression analysis. Multivariate Cox regression analyses were adjusted for sociodemographic and behavioural variables. Regression models were built using stepwise techniques. The $\mathrm{p}$ value $<0.05$ was considered as statistically significant. Statistical analyses were performed using SAS V.9.2 and Joinpoint V.3.4.

\section{RESULTS}

Among the 9412 enrolled MMT clients, 9240 had at least one test for either HIV, HCV or syphilis infections at enrolment and were recruited. Most of the participants included were 30 years or older $(82.12 \%)$, male $(92.50 \%)$ and unemployed $(59.39 \%)$; about half were unmarried (53.92\%); and the majority had low education level (junior high or below: $84.10 \%$ ). The prevalence of HIV, HCV and syphilis at baseline was 8.68\% (768/8851), $75.78 \%(6721 / 8869)$ and $3.11 \%(207 / 6665)$, respectively (see online supplementary table S1). Co-infections of $\mathrm{HIV} / \mathrm{HCV}, \mathrm{HIV} /$ syphilis and HCV/syphilis were found among $5.31 \%$ (452/8512), $0.06 \%$ (4/6667) and $2.37 \%$ $(156 / 6582)$ study participants, respectively, and $0.12 \%$ $(8 / 6667)$ were reportedly infected with all three diseases (see online supplementary figure S1). We identified 4536, 1077 and 3910 individuals who were HIV, HCV and syphilis seronegative at enrolment, respectively, with at least one follow-up test record. Of these, 27, 440 and 98 HIV, HCV and syphilis incidence cases with total follow-up durations of 13360 person-years (pys), 2142 pys and 12727 pys, respectively were reported during the study period (figure 1). In contrast, 3547, 2148 and 2548
HIV, HCV and syphilis-negative participants dropped-out of MMT after the first visit, respectively. Compared with participants included in the analyses, those who dropped out were more likely to be male, less educated, employed, HIV positive but HCV negative and with a shorter history of drug use and less sexual activity in the past 3 months (see online supplementary table S1).

Among the seronegative participants at baseline, follow-up rates at each visit were $51.26 \%, 39.37 \%$, $30.12 \%, 22.15 \%, 14.30 \%, 6.90 \%, 3.15 \%$ for HIV; $81.33 \%$, $48.00 \%, 30.27 \%, 19.87 \%, 12.35 \%, 8.64 \%, 1.86 \%$ for $\mathrm{HCV}$; and $93.27 \%, 74.42 \%, 58.82 \%, 44.73 \%, 31.53 \%$, $16.78 \%, 4.40 \%$ for syphilis, respectively. The overall seroconversion rate of HIV, HCV and syphilis was $0.20(0.13$ to 0.28$) / 100$ pys, 20.54 (18.62 to 22.46$) / 100$ pys, and $0.77(0.62$ to 0.93$) / 100$ pys over the course of MMT, respectively. HIV seroconversion rate declined from $0.17 / 100$ pys at baseline to $0 / 100$ pys among those who remained in treatment after 7 years, corresponding to a moderate but non-significant annual decline of $13.34 \%$ $(-42.48 \%$ to $30.56 \%)$ (Joinpoint regression model; $\mathrm{p}=0.369)$. In comparison, HCV seroconversion rate declined from $24.66 / 100$ pys to $0 / 100$ pys over the same follow-up period, corresponding to a significant annual reduction of $16.12 \%$ ( $5.53 \%$ to $25.52 \%, \mathrm{p}<0.001$ ). Seroconversion rate of syphilis infection $(0.77 / 100$ pys $)$ remained stable over the course of treatment $(\mathrm{p}=0.540)$ (figure $2 \mathrm{~A}$ ).

A positive rate for urine tests was found to be the only predictor for HIV seroconversion $(\geq 60 \%$ vs $<60 \%$; HRs $=3.40,1.07$ to 10.85 ). Being unmarried (HRs $=1.59$, 1.15 to 2.20), injecting drugs in the past 30 days (HRs=2.17, 1.42 to 3.32), having sexual intercourse in the past 3 months (HRs=1.74, 1.22 to 2.47 ) and taking an average MMT dose of over $60 \mathrm{~mL}(\mathrm{HRs}=1.40,1.01$ to 1.94) predicted higher risk of $\mathrm{HCV}$ seroconversion. Participants who were female (HRs=3.56, 2.25 to 5.64) 
Figure 2 (A) HIV, hepatitis C virus $(\mathrm{HCV})$ and syphilis seroconversion rate among drug users over the course of methadone maintenance treatment (MMT). (B) Risk factors associated with HIV, HCV and syphilis seroconversions during the course of MMT, based on multivariate Cox regression.

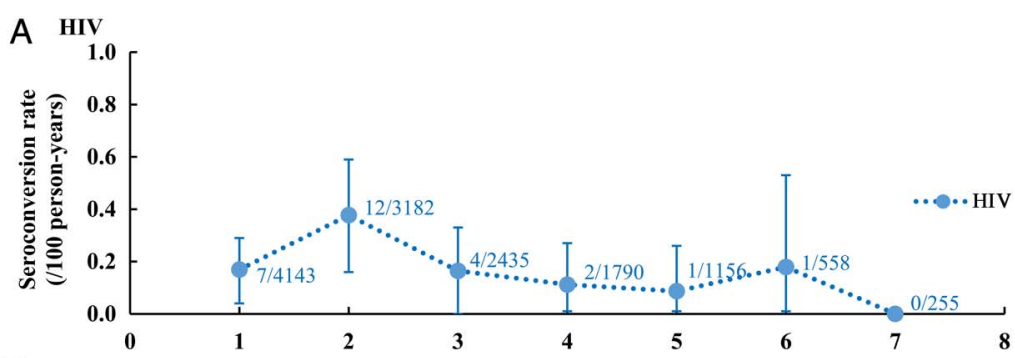

В $\mathrm{HCV}$
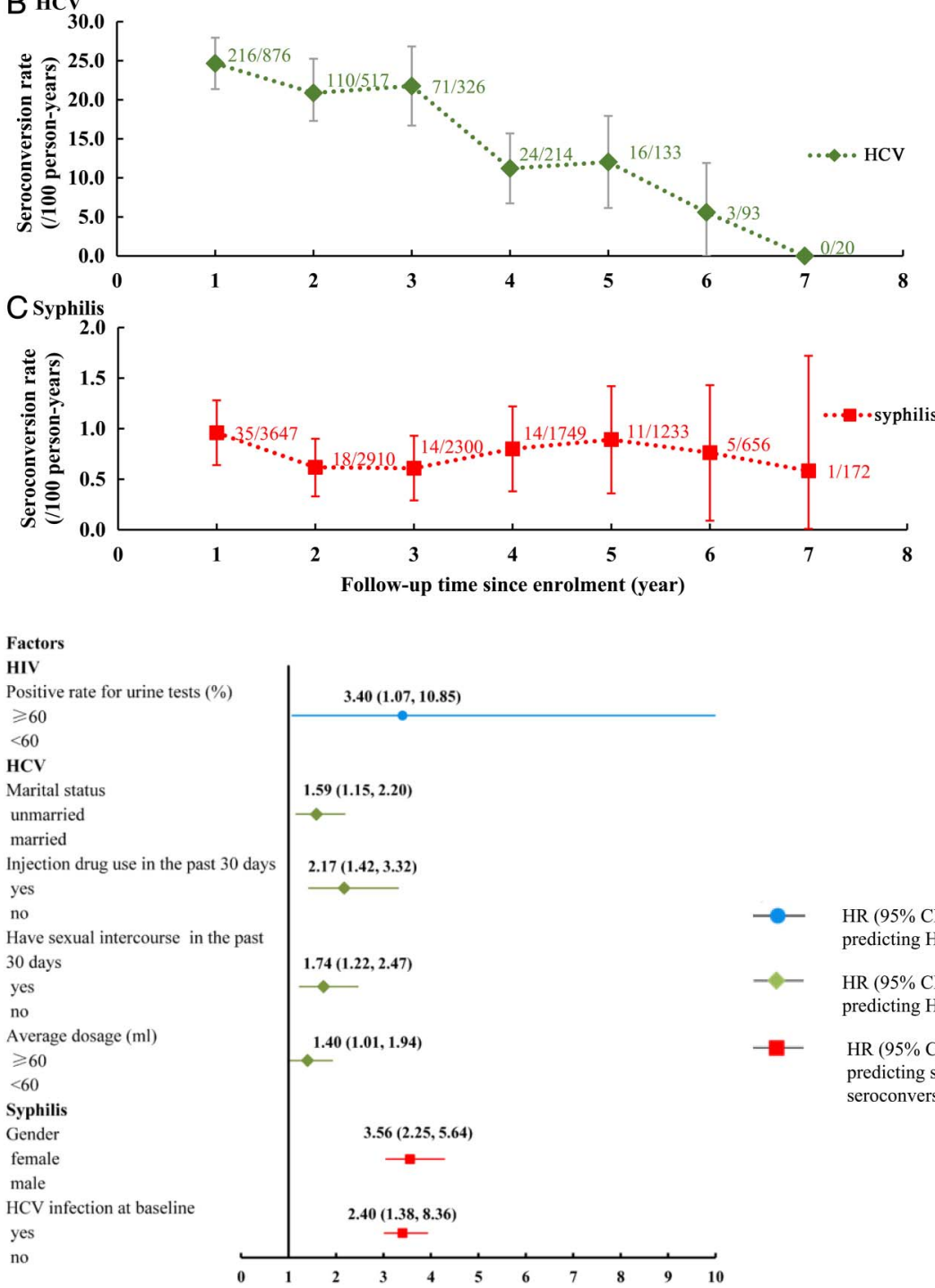

HR $(95 \%$ CI) of risk factors predicting HIV seroconversion HR $(95 \% \mathrm{CI})$ of risk factors predicting HCV seroconversion

HR $(95 \% \mathrm{CI})$ of risk factors predicting syphilis seroconversion and infected with $\mathrm{HCV}$ at baseline (HRs=2.40, 1.38 to 8.36) demonstrated a significantly higher seroconversion rate of syphilis (see online supplementary table S2; and figure 2B).

\section{DISCUSSION}

Our results demonstrated moderate-to-sharp declines in HIV and HCV seroconversion rates among MMT clients over the 7-year follow-up period, while the seroconversion rate of syphilis remained stable. During the course of treatment, seroconversion rates of all three targeted infections (HIV, 0.20/100 pys; syphilis, $0.77 / 100$ pys; and
HCV, 20.54/100 pys) among participants were substantially lower than the corresponding rates among the community of drug users in China (HIV, 0.48-8.01/100 pys; syphilis, 2.41-5.84/100 pys; and HCV, 21.24-39.35/ 100 pys $^{18-22}$ ). The seroconversion rates of HIV, HCV and syphilis among MMT participants in our study were similar to those in other studies in China. ${ }^{16} 2324$

Despite high efficacy in reducing new HCV incidence, MMT is less effective in reducing HIV and syphilis incidence. This is consistent with previous findings. ${ }^{25}$ A plausible explanation may be the disparities in transmission routes and the impacts of MMT on high-risk behaviour. HIV can be transmitted by blood exchange 
and also through sexual contact. In contrast, HCV is readily blood-borne but transmits much less efficiently via sexual intercourse ${ }^{26}$ whereas syphilis predominately transmits through sexual contact. As a harm reduction intervention, MMT is much more effective in reducing injecting behaviours than in reducing unsafe sexual practice. ${ }^{27}$ Previous studies have indicated that the percentage of injecting drug use among Chinese MMT clients dropped dramatically from $81.9 \%$ to $8.1 \%$ within the first 2 years of treatment, whereas the frequency of sexual contacts and proportion of condom use remained largely unvaried. ${ }^{7}$ Biologically, HCV can remain infectious ex vivo much longer than $\mathrm{HIV}^{28}$ and therefore reducing injection practices expectedly results in greater observable decline in seroconversion of HCV than HIV among MMT clients. Given the low seroconversion rate of HIV among MMT participants, our findings suggested that HCV seroconversion rate could be a more sensible biological indicator for assessing the effectiveness of MMT in reducing blood-borne infectious diseases.

Our findings suggest that frequent positive urine test, injecting drug use and high MMT dosage predict higher risks of HIV and HCV seroconversion. Urine testing is a routine monitoring technique for opioid use among MMT clients. A higher proportion of positive urine test results infers more frequent drug use. ${ }^{29}$ Injecting drug use often leads to sharing of injection equipment among drug users and is a well-documented risk factor for HIV and HCV transmission. ${ }^{12}{ }^{15}$ Further, clients with a higher daily methadone dosage may represent drug users with prolonged drug use and more acute addiction. $^{30} 31$ This may imply a subgroup of drug users with high-risk behavioural patterns and treatment difficulties who are hence more prone to the infections. ${ }^{32}$ Notably, recent evidence has suggested that HCV can also be transmitted through sexual contact, ${ }^{33}$ particularly when co-infected with sexually transmitted diseases such as syphilis. Participants who were already married had relatively stable family relationships. In contrast, participants who were unmarried and who had sexual intercourse in the past 30 days had more likelihood of engaging in risky sexual behaviour, thus increasing the risk for HCV infection.

Females are more susceptible to syphilis infection than males, anatomically. ${ }^{34} 35$ In China, a high proportion of female drug users are reportedly involved in commercial sex. ${ }^{14}$ Among them, multiple sexual partnerships and unprotected sexual activities are common. ${ }^{36}$ These risky sexual behaviours increase their risk of syphilis infection. Besides, HCV infection is known to synergise syphilis transmission among drug users, ${ }^{37}$ which contributes a greater likelihood of syphilis acquisition.

The present study has several limitations. First, frequency of HIV, HCV and syphilis testing was every 6 months. Similar to other studies, the estimated timing of seroconversion relied on the consecutive measurements of negative and positive testing results, rather than a biological test for recent infection. Second, individuals with certain characteristics, especially those with high-risk behaviour, are more likely to drop-out from MMT; exclusion of this subgroup leads to underestimation of the actual seroconversion rates. Third, the percentage of drug use may be under-reported as study participants may tend to provide socially desirable responses to surveys. Fourth, due to the scarce number of HIV seroconverted cases, only one significant independent variable has been found to predict HIV seroconversion.

The finding that MMT effectively reduces seroconversion of HIV and HCV, highlights the importance of improving programme coverage and client retention of MMT in China. Integration of effective HIV intervention strategies, such as needle and syringe exchange, and condom distribution programmes, should be promoted to reduce high-risk behaviours at the early stage of treatment. Although MMT clinics provide free condoms to clients, the proportion of condom use among MMT clients is reportedly low in China. ${ }^{38}$ Low perception of risks for blood-borne and sexually transmitted infections is a major barrier for health service utilisation. ${ }^{39}$ Condom programmes should be integrated as one of the auxiliary services in MMT clinics. Health education and counselling services regarding disease prevention and treatment targeting clients, particularly those at high risk, should also be routinely provided in MMT clinics in China. China has actively responded to the HIV epidemic and substantial achievements have been made in the last decade. Antiretroviral treatment is provided freely to eligible HIV-positive individuals, but treatment for HCV and syphilis may incur substantial financial burdens for drug users. Given the currently high cost of direct-acting antiviral agents for HCV infection, its treatment is unlikely to be provided free to the needy in the near future. Although MMT clinics offer treatment referral for positive HCV and syphilis diagnoses, few clients can afford the medical cost for treatment, resulting in delay and missed opportunities for timely treatment. ${ }^{40} \mathrm{In}$ the absence of a national treatment programme, the MMT programme could thus be a feasible alternative for preventing $\mathrm{HCV}$ transmission among drug users. Further, linking antiretroviral treatment to MMT may reduce mortality among drug users living with HIV. ${ }^{41}$ With the new development of a HCV cure, ${ }^{42}$ expansion of HCV treatment for MMT clients may be considered a priority.

Acknowledgements The authors acknowledge all the staff of the MMT clinics in Guangdong Province, China.

Contributors LL, LZ and XZ conceived the study. LL contributed to study coordination. XZ participated in data collection and analysis, and drafted the manuscript. $L Z$ and $L L$ finalised the manuscript. All the authors read and approved the final manuscript.

Funding This work was supported by the National Natural Science Foundation of China (grant 30972552, grant 71173245, grant 81473065) and the China Medical Board (grant 12-111).

Competing interests None declared.

Patient consent Obtained. 
Ethics approval The Sun Yat-sen University Ethics Committee granted ethical approval ((2013) Ref No 26) for the study.

Provenance and peer review Not commissioned; externally peer reviewed.

Data sharing statement No additional data are available.

Open Access This is an Open Access article distributed in accordance with the Creative Commons Attribution Non Commercial (CC BY-NC 4.0) license, which permits others to distribute, remix, adapt, build upon this work noncommercially, and license their derivative works on different terms, provided the original work is properly cited and the use is non-commercial. See: http:// creativecommons.org/licenses/by-nc/4.0/

\section{REFERENCES}

1. WHO. Injecting drug use. World Health Organization, 2014.

2. UNODC. World Drug Report 2014. New York, 2014.

3. Nelson PK, Mathers BM, Cowie B, et al. Global epidemiology of hepatitis $B$ and hepatitis $C$ in people who inject drugs: results of systematic reviews. Lancet 2011;378:571-83.

4. Todd CS, Nasir A, Raza Stanekzai M, et al. Prevalence and correlates of syphilis and condom use among male injection drug users in four Afghan cities. Sex Transm Dis 2010;37:719-25.

5. Shi J, Zhao LY, Epstein DH, et al. The effect of methadone maintenance on illicit opioid use, human immunodeficiency virus and hepatitis $C$ virus infection, health status, employment, and criminal activity among heroin abusers during 6 months of treatment in china $J$ Addict Med 2007;1:186-90.

6. Security NCBotMoP. Annual report on drug control in China 2014. Beijing, 2014

7. Zhou Y, Li X, Zhang C, et al. Rates of HIV, syphilis, and HCV infections among different demographic groups of female sex workers in Guangxi China: evidence from 2010 national sentinel surveillance data. AIDS Care 2013;25:1433-41.

8. Duan S, Yang YC, Han J, et al. [Study on incidence of HIV infection among heroin addicts receiving methadone maintenance treatment in Dehong prefecture, Yunnan province]. Zhonghua Liu Xing Bing Xue Za Zhi 2011;32:1227-31.

9. Peles E, Schreiber S, Rados V, et al. Low risk for hepatitis C seroconversion in methadone maintenance treatment. J Addict Med 2011;5:214-20.

10. Tsui JI, Evans JL, Lum PJ, et al. Association of opioid agonist therapy with lower incidence of hepatitis $C$ virus infection in young adult injection drug users. JAMA Intern Med 2014;174:1974-81.

11. Zhuang X, Liang Y, Chow EP, et al. HIV and HCV prevalence among entrants to methadone maintenance treatment clinics in China: a systematic review and meta-analysis. BMC Infect Dis 2012;12:130.

12. Xiaoli W, Lirong W, Xueliang W, et al. Risk factors of hepatitis C virus infection in drug users from eleven methadone maintenance treatment clinics in Xi'an, China. Hepat Mon 2014;14:e19601.

13. Broers $B$, Junet $C$, Bourquin $M$, et al. Prevalence and incidence rate of $\mathrm{HIV}$, hepatitis $\mathrm{B}$ and $\mathrm{C}$ among drug users on methadone maintenance treatment in Geneva between 1988 and 1995. AIDS 1998;12:2059-66.

14. Gourevitch MN, Hartel D, Schoenbaum EE, et al. A prospective study of syphilis and HIV infection among injection drug users receiving methadone in the Bronx, NY. Am J Public Health 1996;86:1112-15.

15. Zhuang $X$, Wang $Y$, Chow EP, et al. Risk factors associated with $\mathrm{HIV/HCV}$ infection among entrants in methadone maintenance treatment clinics in China: a systematic review and meta-analysis. Drug Alcohol Depend 2012;126:286-95.

16. Zou X, Chen W, Ling L. Influencing factors of HIV and syphilis seroconversion among patients with methadone maintenance treatment. Chin J Public Health 2013;29:1107-11.

17. Yu B, Barrett MJ, Kim HJ, et al. Estimating Joinpoints in continuous time scale for multiple change-point models. Comput Stat Data Anal 2007:51:2420-7.

18. Li X. A research of the incidence of HIV/HCV/HBV/syphilis and risk behaviors among injecting drug users in Xichang County of Sichuan Province. Suzhou University, 2011.

19. Bruneau J, Roy E, Arruda N, et al. The rising prevalence of prescription opioid injection and its association with hepatitis $C$ incidence among street-drug users. Addiction 2012;107:1318-27.
20. van den Berg $\mathrm{CH}$, Smit C, Bakker M, et al. Major decline of hepatitis $C$ virus incidence rate over two decades in a cohort of drug users. Eur J Epidemiol 2007;22:183-93.

21. Duan S, Han J, Tang RH, et al. [Study on the incidence and risk factors of HCV infection among heroin addicts who were on methadone maintenance treatment in Dehong prefecture, Yunnan province]. Zhonghua Liu Xing Bing Xue Za Zhi 2013;34:552-6.

22. Sarna A, Saraswati LR, Sebastian M, et al. High HIV incidence in a cohort of male injection drug users in Delhi, India. Drug Alcohol Depend 2014;139:106-14.

23. Zhou W, Wang $X$, Zhou $S$, et al. Hepatitis $C$ seroconversion in methadone maintenance treatment programs in Wuhan, China Addiction 2015;110:796-802.

24. Chang YP, Duo L, Kumar AMV, et al. Retention and HIV seroconversion among drug users on methadone maintenance treatment in Yunnan, China. Public Health Action 2014;4:28-34.

25. Ruan $\mathrm{Y}$, Liang $\mathrm{S}$, Zhu J, et al. Evaluation of harm reduction programs on seroincidence of HIV, hepatitis B and C, and syphilis among intravenous drug users in southwest China. Sex Transm Dis 2013;40:323-8.

26. Garfein RS, Vlahov D, Galai N, et al. Viral infections in short-term injection drug users: the prevalence of the hepatitis $\mathrm{C}$, hepatitis $\mathrm{B}$, human immunodeficiency, and human T-lymphotropic viruses. $A m \mathrm{~J}$ Public Health 1996;86:655-61.

27. Marsch LA. The efficacy of methadone maintenance interventions in reducing illicit opiate use, HIV risk behavior and criminality: a metaanalysis. Addiction 1998;93:515-32.

28. Crofts N, Dore G, Locarnini S. Hepatitis C: an Australian perspective. Melbourne, 2001.

29. Pergolizzi J, Pappagallo M, Stauffer J, et al., Integrated Drug Compliance Study Group (IDCSG). The role of urine drug testing for patients on opioid therapy. Pain Pract 2010;10:497-507.

30. $\mathrm{Li} \mathrm{X}$, Tan H, Sun Z, et al. [Change and influence factors of craving for patients after 6 month methadone maintenance treatment]. Zhong Nan Da Xue Xue Bao Yi Xue Ban 2009;34:718-22.

31. Baumeister M, Vogel M, Dürsteler-MacFarland K, et al. Association between methadone dose and concomitant cocaine use in methadone maintenance treatment: a register-based study. Subst Abuse Treat Prev Policy 2014;9:1-8.

32. Xia X, Luo J, Bai J, et al. Epidemiology of hepatitis $\mathrm{C}$ virus infection among injection drug users in China: systematic review and meta-analysis. Public Health 2008;122:990-1003.

33. Terrault NA, Dodge JL, Murphy EL, et al. Sexual transmission of hepatitis $C$ virus among monogamous heterosexual couples: the HCV partners study. Hepatology 2013;57:881-9.

34. Ruan Y, Liang S, Zhu J, et al. Gender and ethnic disparities of HIV and syphilis seroconversions in a 4-year cohort of injection drug users. Southeast Asian J Trop Med Public Health 2013;44:842-53.

35. Coffin LS, Newberry A, Hagan $\mathrm{H}$, et al. Syphilis in drug users in low and middle income countries. Int J Drug Policy 2010;21:20-7.

36. Folch C, Casabona J, Espelt A, et al., REDAN Study Group. Gender differences in HIV risk behaviours among intravenous drug users in Catalonia, Spain. Gac Sanit 2013;27:338-43.

37. Liao M, Kang D, Tao X, et al. Syndemics of syphilis, HCV infection, and methamphetamine use along the east coast of China. BMC Public Health 2014;14:172.

38. Xue H, Duo L, Lei Y, et al. Cohort analysis of change in high risk behavior among people received methadone maintenance treatment. Chin J Drug Depend 2013;22:460-3.

39. Du J, Wang Z, Xie B, et al. Hepatitis C knowledge and alcohol consumption among patients receiving methadone maintenance treatment in Shanghai, China. Am J Drug Alcohol Abuse 2012;38:228-32.

40. Zhao Y, Shi CX, McGoogan JM, et al. Predictors of accessing antiretroviral therapy among HIV-positive drug users in China's National Methadone Maintenance Treatment Programme. Addiction 2015;110(Suppl 1):40-50.

41. Cao X, Wu Z, Li L, et al. Mortality among methadone maintenance clients in China: a six-year cohort study. PLOS ONE 2013;8:e82476.

42. Manns M, Pol S, Jacobson IM, et al., HALLMARK-DUAL Study Team. All-oral daclatasvir plus asunaprevir for hepatitis $C$ virus genotype $1 \mathrm{~b}$ : a multinational, phase 3 , multicohort study. Lancet 2014;384:1597-605. 